OPEN ACCESS

Edited by:

Van-Tuan Tran,

Vietnam National University, Hanoi,

Vietnam

Reviewed by:

Zhilong Wang,

Shanghai Jiao Tong University, China

Chan Zhang,

Beijing Technology and Business

University, China

Yanping Li,

Nanchang University, China

${ }^{*}$ Correspondence:

$\mathrm{LiNi}$

nili@fzu.edu.cn

Xucong LV

xucong1154@163.com

Specialty section:

This article was submitted to

Food Microbiology,

a section of the journal

Frontiers in Microbiology

Received: 30 April 2021

Accepted: 25 May 2021

Published: 21 June 2021

Citation:

Zhou K, Wu L, Chen G, Liu Z, Zhao $X$, Zhang $C, L v X$, Zhang $W$, Rao $P$ and Ni L (2021) Development

of a Novel Restrictive Medium

for Monascus Enrichment From Hongqu Based on the Synergistic Stress of Lactic Acid and Ethanol.

Front. Microbiol. 12:702951. doi: 10.3389/fmicb.2021.702951

\section{Development of a Novel Restrictive Medium for Monascus Enrichment From Hongqu Based on the Synergistic Stress of Lactic Acid and Ethanol}

\author{
Kangxi Zhou',2, Li Wu'2, Guimei Chen'2, Zhibin Liu'2, Xinze Zhao'2, Chen Zhang², \\ Xucong Lv' ${ }^{2 *}$, Wen Zhang ${ }^{2}$, Pingfan $\mathrm{RaO}^{2}$ and $\mathrm{Li} \mathrm{Ni}^{1,2 *}$ \\ ${ }^{1}$ College of Chemical Engineering, Fuzhou University, Fuzhou, China, ${ }^{2}$ Fujian Center of Excellence for Food Biotechnology, \\ Institute of Food Science and Technology, Fuzhou University, Fuzhou, China
}

Hongqu is a famous fermented food produced by Monascus and has been used as food coloring, wine starters and food additives for thousands of years in China. Excellent Monascus strain is an important prerequisite for producing high-quality Hongqu. However, the isolation of Monascus pure culture from Hongqu samples is time-consuming and laborious because it is easily interfered by other microorganisms (especially filamentous fungi). Therefore, the development of restrictive medium for Monascus enrichment from Hongqu is of great significance for the preparation and screening of excellent Monascus strains. Results of this study showed that Monascus has good tolerance to lactic acid and ethanol. Under the conditions of tolerance limits [7.5\% lactic acid (v/v) and $12.0 \%$ ethanol ( $/ \mathrm{v})$ ], Monascus could not grow but it still retained the vitality of spore germination, and the spore activity gradually decreased with the increasing concentrations of lactic acid and ethanol. More interestingly, the addition of lactic acid and ethanol significantly changed the microbial community structure in rice milk inoculated with Hongqu. After response surface optimization, Monascus could be successfully enriched without the interference of other microorganisms when 3.98\% $(\mathrm{v} / \mathrm{v})$ lactic acid and $6.24 \%(\mathrm{v} / \mathrm{v})$ ethanol were added to rice milk simultaneously. The optimal enrichment duration of Monascus by the restrictive medium based on the synergistic stress of lactic acid and ethanol is $8 \sim 24 \mathrm{~h}$. The synergistic stress of lactic acid and ethanol had no obvious effects on the accumulation of major metabolites in the progeny of Monascus, and was suitable for the enrichment of Monascus from different types of Hongqu. Finally, the possible mechanisms on the tolerance of Monascus to the synergistic stress of lactic acid and ethanol were preliminarily studied. Under the synergistic stress of lactic acid and ethanol, the cell membrane of Monascus defends against lactic acid and ethanol into cells to some extent, and the superoxide dismutase (SOD), catalase (CAT) and glutathione peroxidase (GSH-Px) activities of Monascus were higher than those of other fungi, which significantly reduced the degree of lipid peroxidation of cell membrane, while secreting more amylase to make reducing sugars to provide the cells with enough energy to resist environmental stress. This work has great application value for the construction of Monascus strain library and the better development of its germplasm resources.

Keywords: Monascus enrichment, restrictive medium, Hongqu, lactic acid, ethanol 


\section{INTRODUCTION}

Hongqu, also called red fermented rice, red yeast rice and Anka, is produced from steamed rice inoculated with Monascus seed culture through solid-state fermentation (Liu et al., 2018). It has been used for thousands of years in Asian countries and is currently used worldwide as a food coloring, fermentation starter and food additives (Tian et al., 2016; Lei et al., 2019). Monascus, an essential microorganism for the production of Hongqu, can metabolize a variety of useful enzymes and secondary metabolites (Zhou et al., 2019). For example, liquefying enzymes and saccharifying enzymes contribute to the degradation of starch in grains and the formation of reducing sugars (Shin et al., 2017; Ai et al., 2019); Monascus pigment can be used for food coloring and antisepsis (Agboyibor et al., 2018); and lovastatin and $\gamma$-aminobutyric acid are beneficial to the development of lipid-lowering and blood pressure-reducing drugs, respectively (Eren et al., 2015; Zhang et al., 2018; Xiong et al., 2019). To fully understand the production and application values of Monascus, it is necessary to isolate and purify the original strain from Hongqu to obtain high-quality Monascus.

Most Hongqu production follows the manual fermentation techniques of ancient China, so the production process of Hongqu is not strictly controlled (Park et al., 2016; Huang et al., 2019). In addition to Monascus, bacteria such as Lactobacillus and Bacillus and fungi such as yeast, Aspergillus niger, and Aspergillus oryzae may be present in Hongqu (Lv et al., 2015, 2016). The presence of these microorganisms can cause serious disturbance during the preparation of Monascus pure culture from Hongqu. Most of the purification of Monascus does not involve exclusive pretreatment and involves direct coating or streaking on mold medium. The media used in these methods include conventional mold media such as potato dextrose agar (Lv et al., 2016), Rose Bengal medium (Huang et al., 2014), malt extract agar (Barbosa et al., 2017) and mold media supplemented with antibiotics or sodium deoxycholate (Zhang et al., 2015). Although these methods are convenient to operate, they cannot exclude the interference of fungi other than Monascus. The spores of various microorganisms often diffuse in the culture dish, and repeated operations are required to obtain Monascus. Therefore, it is necessary to invent a novel restrictive medium for the enrichment of Monascus.

To enrich all kinds of Monascus as much as possible, the type and concentration of regulatory factors for purification should meet the following requirements: other microorganisms are eliminated without inactivation of Monascus. Thus, Monascus must be well tolerated with these regulatory factors. Monascus has been reported to have good tolerance characteristics toward lactic acid and ethanol. For example, Monascus ruber CBS 127564 can tolerate $14.5 \%$ (v/v) lactic acid (Weusthuis et al., 2017), and Monascus purpureus FJMR24 can tolerate 18\% (v/v) ethanol (Ren et al., 2021). However, although it is not uncommon for microorganisms to tolerate lactic acid or ethanol, just as Terrilactibacillus laevilacticus SK5-6 can tolerate approximately $12 \%$ (v/v) lactic acid (Prasirtsak et al., 2017) and Candida tropicalis can tolerate approximately $30 \%(\mathrm{v} / \mathrm{v})$ ethanol (Balia et al., 2018), there are very few reports on non-genetically modified microorganisms that can tolerate high concentrations of lactic acid and ethanol simultaneously. Monascus has the potential to tolerate lactic acid and ethanol simultaneously, so it is expected to be enriched from Hongqu through a restrictive medium based on the synergistic stress of lactic acid and ethanol.

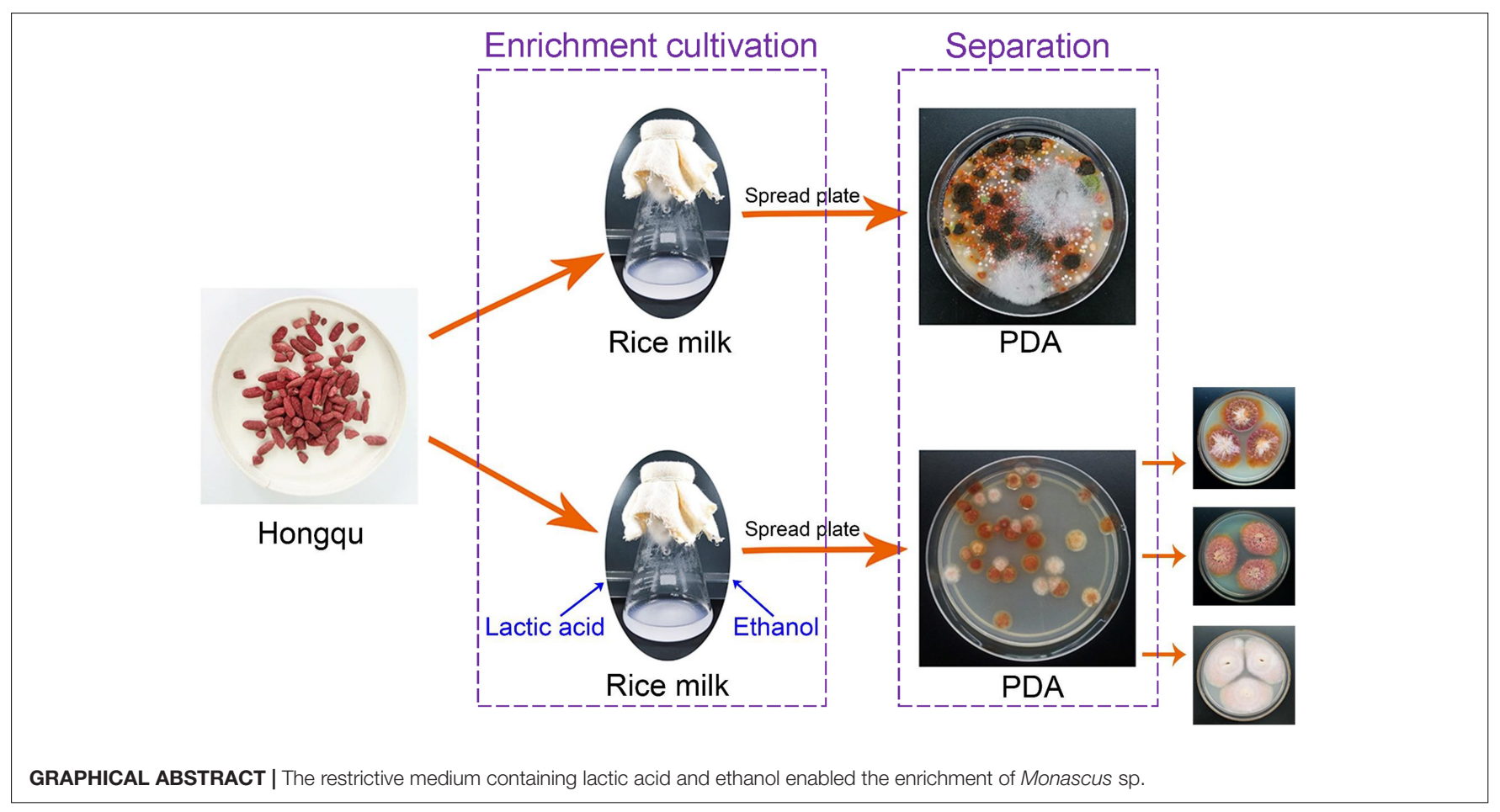


In this study, we explored the possibility of lactic acid and ethanol as regulatory factors of a restrictive medium for Monascus enrichment from Hongqu, and also preliminarily speculated the possible mechanisms on the tolerance of Monascus to the synergistic stress of lactic acid and ethanol. These results would help to improve the efficiency of Monascus enrichment from Hongqu and provides a reference for better mining of Monascus germplasm resources.

\section{MATERIALS AND METHODS}

\section{Sources of Monascus and Hongqu}

$M$. serorubescens A1, M. lunisporas A4, M. sanguineus B1, $M$. kaoliang B6, M. argentinensis B7, M. purpureus C1 was isolated from Hongqu by our laboratory (Lv et al., 2012; Cai et al., 2019). Nucleotide sequences for ITS-5.8S rDNA region of these Monascus were deposited in the NCBI database under accession numbers MZ297243-MZ297247 and MZ297277 respectively. Hongqu samples used in this study were collected from different cities in Fujian Province, China: BHQ26 (Hongqu for rice wine brewing, from Ningde city), BHQ33 (Hongqu for rice wine brewing, from Quanzhou city), PHQ30 (Hongqu for pigment production, from Ningde city), WHQ38 (Wuyi Hongqu, a special Hongqu for rice wine brewing that contains more Aspergillus niger than other Hongqu, from Fuzhou city).

\section{Mediums for Microbial Culture and Fermentation}

Potato dextrose agar (PDA) (Guangdong Huankai Microbial Sci. \& Tech. Co., Ltd., Guangzhou, China) was configured according to the product manual. The modified PDA medium was prepared by adding $0.1 \mathrm{~g} / \mathrm{L}$ chloramphenicol and $0.5 \mathrm{~g} / \mathrm{L}$ sodium deoxycholate to the original PDA. The modified potato dextrose water (PD) medium is based on PD medium (Guangdong Huankai Microbial Sci. \& Tech. Co., Ltd., Guangzhou, China) with $15 \mathrm{~g} / \mathrm{L}$ indica rice flour, $10 \mathrm{~g} / \mathrm{L}$ sodium glutamate, and $5 \mathrm{~g} / \mathrm{L}$ glycerol. The rice milk containing $15 \mathrm{~g} / \mathrm{L}$ indica rice flow, $5 \mathrm{~g} / \mathrm{L}$ glucose and $0.1 \mathrm{~g} / \mathrm{L}$ chloramphenicol was used as the basic medium, and different contents of hydrochloric acid ( $\mathrm{HCl})$, lactic acid and ethanol were added to this rice milk as the restrictive medium according to the experimental requirements.

\section{Tolerance Test of Monascus Against Lactic Acid and Ethanol}

All the Monascus strains were activated on PDA plates and incubated at $30^{\circ} \mathrm{C}$ for 7 days under aerobic conditions. Spores were harvested by flooding the surface of the agar with normal saline. The spore suspension was adjusted with sterile saline solution to $10^{6} \mathrm{CFU} / \mathrm{mL}$ with a hemocytometer. The Monascus spore suspension was connected to different restrictive medium at $1 \%(\mathrm{v} / \mathrm{v})$ inoculum size and cultured for 7 days. After the fermentation, samples were taken to determine the viable number and biomass increment of Monascus.

\section{Separation of Monascus Pure Culture From Hongqu}

Hongqu was ground into Hongqu powder by using a sterile mortar. Direct dilution coating method: $0.1500 \pm 0.0010 \mathrm{~g}$ Hongqu powder was accurately weighed and put into $30 \mathrm{~mL}$ sterile saline solution and shake for $90 \mathrm{~s}$. The insoluble particles were filtered out with four layers of gauze and the remaining liquid containing microbial spores diluted and coated on the modified PDA medium. Fermentation-coating method: $0.1500 \pm 0.0010 \mathrm{~g}$ Hongqu powder was accurately weighed and fermented in a $250 \mathrm{~mL}$ shake flask containing $30 \mathrm{~mL}$ restrictive medium at $30^{\circ} \mathrm{C}$ and $200 \mathrm{r} / \mathrm{min}$. After the fermentation, the fermentation broth was diluted and coated on the modified PDA medium for 4 days at $30^{\circ} \mathrm{C}$.

\section{Effects of Lactic Acid and Ethanol on Morphology and Metabolism of Purified Monascus Progeny}

The spore suspension of Monascus A1, A4, B1, B6, B7, C1 were respectively inoculated into the restrictive medium containing $3.98 \%(\mathrm{v} / \mathrm{v})$ lactic acid and $6.24 \%(\mathrm{v} / \mathrm{v})$ ethanol for 7 days at $30^{\circ} \mathrm{C}$ and $200 \mathrm{r} / \mathrm{min}$. The fermentation broth of the restrictive medium was used as the treatment group, and the spore suspension harvested from the PDA plate was used as the control group. Each group contained 6 Monascus species. The two groups of Monascus have activated to PDA plates again and then spotted on the modified PDA for 7 days at $30^{\circ} \mathrm{C}$ to observe the colony morphology, at the same time, fermented in a $250 \mathrm{~mL}$ shake flask containing $30 \mathrm{~mL}$ modified PD medium at $30^{\circ} \mathrm{C}$ and $200 \mathrm{r} / \mathrm{min}$ for 7 days to detect metabolites.

\section{Metabolic Behaviors of Fungus Isolated From BHQ33 Against Lactic and Ethanol Stress}

All the fungus isolated from BHQ33 were activated to PDA plates and incubated at $30^{\circ} \mathrm{C}$ for 7 days under aerobic conditions. Spores were harvested by flooding the surface of the agar with sterile saline solution and were adjusted with normal saline to $10^{6} \mathrm{CFU} / \mathrm{mL}$ with a hemocytometer. The above fungal spore suspensions were first respectively inoculated at $1 \%(\mathrm{v} / \mathrm{v})$ inoculum size into basal medium (rice milk, containing $15 \mathrm{~g} / \mathrm{L}$ indica rice flour, $5 \mathrm{~g} / \mathrm{L}$ glucose, and $0.1 \mathrm{~g} / \mathrm{L}$ chloramphenicol) for adaptive growth at $30^{\circ} \mathrm{C}$ and $200 \mathrm{r} / \mathrm{min}$ for 7 days, followed by centrifugation at $3,000 \mathrm{r} / \mathrm{min}$ for $15 \mathrm{~min}$ to collect the cells, which were then washed 3 times with sterile saline solution. The washed cells were resuspended to $10 \mathrm{mg} / \mathrm{mL}$ with sterile saline solution and were connected to different selective media at $10 \%$ (v/v) inoculum size and cultured for $24 \mathrm{~h}$. There are four kinds of selective media in this part experiment: basal medium without lactate and ethanol (control), basal medium supplemented with $3.98 \%(\mathrm{v} / \mathrm{v})$ lactic acid (experimental group 1), basal medium supplemented with $6.24 \%$ (v/v) ethanol (experimental group 2) and basal medium supplemented with both $3.98 \%(\mathrm{v} / \mathrm{v})$ lactic acid and $6.24 \%(\mathrm{v} / \mathrm{v})$ ethanol (experimental group 3). 


\section{Analysis of Fungi Quantity}

In the tolerance test of Monascus, the plate count and glucosamine content of biomass were used to analyze the biomass increment of Monascus in different selective media, and the plate count method was used to determine the number of viable fungi in the samples in other experiments. Glucosamine is the main component of chitin in Monascus cell wall, and its content is often used to predict the biomass of Monascus in samples containing indica rice. With the dry cell of Monascus as a standard reference, the standard curve between the absorbance value and the dry weight of the cell was drawn after four steps of acidolysis, neutralization, Elson-Morgan reaction and colorimetric determination (Sakural et al., 1977; Chysirichote et al., 2013). The sample was converted into biomass based on the standard curve.

\section{Identification of Isolated Fungal Strains}

The fungi isolated and purified from the modified PDA medium were identified by DNA analysis: ITS- $5.8 \mathrm{~S}$ rDNA region was partially amplified and sequenced using primers ITS1/ITS4 as described by Park et al. (2004). Each $25 \mu \mathrm{L}$ PCR reaction mixture consisted of $1 \mu \mathrm{L}$ DNA template, $12.5 \mu \mathrm{L} 2 \times$ Power Taq PCR MasterMix (containing $\mathrm{MgCl}_{2}$ ) (Sangon Biotech Co., Ltd. Shanghai, China), $1 \mu \mathrm{L}$ primer, and $\mathrm{ddH}_{2} \mathrm{O}$ was added to a final volume of $25 \mu \mathrm{L}$. The PCR was conducted under the following conditions: initial denaturation at $95^{\circ} \mathrm{C}$ for $5 \mathrm{~min}$, 35 cycles of denaturation at $94^{\circ} \mathrm{C}$ for $45 \mathrm{~s}$, annealing at $55^{\circ} \mathrm{C}$ for $45 \mathrm{~s}$, extension at $72^{\circ} \mathrm{C}$ for $120 \mathrm{~s}$, followed by a final extension cycle at $72^{\circ} \mathrm{C}$ for $10 \mathrm{~min}$ prior to maintaining the mixture at $4^{\circ} \mathrm{C}$. Products were analyzed on $1.5 \%$ agarose gel containing $0.1 \mu \mathrm{L} / \mathrm{mL}$ of $4 \mathrm{~S}$ Red Plus nucleic acid stain (Sangon Biotech Co., Ltd. Shanghai, China) and visualized under UV light (UV source GelDoc 1000, Bio-Rad). PCR products were gel-purified with GFX ${ }^{\mathrm{TM}}$ PCR DNA and Gel Band Purification Kit (Amersham Biosciences AB, Uppsala), according to the manufacturer's instructions. Purified PCR products were directly sequenced using the $\mathrm{ABI}$ prism 3730 DNA analyzer (Applied Biosystems, Foster). Sequences were analyzed using Blast at NCBI. ${ }^{1}$ Nucleotide sequences for ITS5.8S rDNA region of isolates represent Monascus purpureus BHQ33.M01, Monascus purpureus BHQ33.M02, Monascus purpureus BHQ33.M03, Saccharomyces cerevisiae BHQ33.S01, Aspergillus niger BHQ33.AN01, Lichtheimia ramosa BHQ33.L01, Trichoderma BHQ33.T01, and Aspergillus flavus BHQ33.AF01 were deposited in the NCBI database under accession numbers MW581230-MW581237.

\section{Analysis of Metabolites in the Regenerated Progenies of Monascus}

The analytical method of $\gamma$-aminobutyric acid was referenced to the agricultural industry-standard NY/T 2890-2016 of the people's Republic of China, unit: $\mu \mathrm{g} / \mathrm{mL}$. The pigment, lovastatin and citrinin were extracted with $70 \%(\mathrm{v} / \mathrm{v})$ ethanol at $60^{\circ} \mathrm{C}$ for $2 \mathrm{~h}$. The mycelium of the sample was removed by centrifugation

${ }^{1}$ http://www.ncbi.nlm.nih.gov/ at 5,000 r/min for $10 \mathrm{~min}$, and the supernatant was diluted and filtered through a $0.22 \mu \mathrm{m}$ filter membrane to be tested. The content of Monascus pigment was predicted by the absorbance value using a spectrophotometer (U1900, Hitachi, Japan) at $505 \mathrm{~nm}$ referred to the national standards of the people's Republic of China GB 1886.19-2015, unit: U/mL; The HPLC (L2000, Hitachi, Japan) analysis method of lovastatin referred to the light industry standard of the people's Republic of China GB/T 28472007, unit: $\mu \mathrm{g} / \mathrm{mL}$; The HPLC analysis method of citrinin refers to the national standard of the people's Republic of China GB 5009.222-2016, unit: $\mathrm{ng} / \mathrm{mL}$.

The amylase activity was measured using an $\alpha$-amylase assay kit (all kits used in the current study were purchased from Nanjing Jiancheng Bioengineering Institute, Nanjing, China, unless otherwise indicated) based on QB/T 4257-2011. One unit of amylase activity was defined as the quantity of enzyme metabolized by $1 \mathrm{~g}$ mycelium that was required to hydrolyze $1 \mathrm{mg}$ soluble starch per hour, at $35^{\circ} \mathrm{C}$ and $\mathrm{pH} 4.6$. The glucoamylase activity was measured according to the Light Industry Standard of the People's Republic of China QB/T 4257-2011 with slight modifications. One unit of glucoamylase was defined as the amount of enzyme metabolized by $1 \mathrm{~g}$ mycelia that was required to release $1 \mathrm{mg}$ glucose per hour from soluble starch, at $35^{\circ} \mathrm{C}$ and $\mathrm{pH}$ 4.6.

\section{Metabolic Behaviors of Fungus Isolated From BHQ33 Against Lactic Acid and Ethanol Stress}

The fermented medium was diluted to $10 \mathrm{mg} / \mathrm{mL}$ with sterile saline solution. $20 \mathrm{~mL}$ aliquot of the sample was centrifuged at 3,000 r/min for $15 \mathrm{~min}$, and the supernatant, which had been filtered through a $0.45 \mu \mathrm{m}$ filter, was used as a sample to be tested for extracellular metabolites. The centrifuged cells were washed 3 times with sterile saline solution and resuspended to $15 \mathrm{~mL}$, followed by ultrasonic disruption (FS-200T, SXSONIC, China) at $200 \mathrm{~W}$ for $30 \mathrm{~min}$ under ice bath conditions. The ultrasonic fragmentation cycle times were as follows: $5 \mathrm{~s}$ working, 5 s stagnating, and $20 \mathrm{kHz}$ frequency. After disruption, cell debris was removed by centrifugation at 4,500 r/min for $20 \mathrm{~min}$, and the supernatant was used as the sample to be tested for intracellular metabolites.

The analytical methods for lactic acid and ethanol were referred to previous studies by Jiang et al. (2019), and Cai et al. (2019), respectively. The reducing sugars were determined by 3, 5-dinitrosalicylicacid method (Xu et al., 2014). Protein concentrations were measured using a coomassie (Bradford) protein assay kit. Soluble starch content and amylase activity were measured using an $\alpha$-amylase assay kit with some modifications. In detail, soluble starch content is measured by the chromogenic reaction of the iodide solution with the extracellular solution, and the definition of one unit of amylase activity in this system was modified as the quantity of enzyme metabolized by $1 \mathrm{~mL}$ extracellular solution that was required to hydrolyze $1 \mathrm{mg}$ soluble starch per hour, at $35^{\circ} \mathrm{C}$ and $\mathrm{pH}$ 4.6. Total superoxide dismutase (SOD), malondialdehyde (MDA), catalase (CAT), and total glutathione peroxidase (GSH-Px) levels of intracellular 
metabolism were measured with the assay kits according to the manufacturer's instructions (Nanjing Jiancheng Institute of Biotechnology, China).

\section{Statistical Analysis}

The experiments were conducted in triplicate, and the results were presented as the mean \pm standard deviation (SD). One-way analysis of variance (ANOVA) was performed across multiple groups to test significance $(p<0.05)$ using SPSS 23.0 software (SPSS Inc., Chicago, United States). A histogram was generated using Origin 2018 software (OriginLab, Northampton, MA, United States). Response surface design (RSD) was carried out by Design-Expert V8.0.6.1software (Stat-Ease, Inc., Minneapolis, $\mathrm{MN}$, United States).

\section{RESULTS}

\section{Tolerance of Monascus to Lactic Acid and Ethanol}

The range of common tolerance of Monascus to growth regulatory factors can be roughly determined by tolerance tests with multiple species Monascus, which facilitates selection of the type and concentrations of growth regulatory factors added to the restrictive medium. Monascus can grow in the medium containing low concentrations of lactic acid and ethanol with a certain number of live spores (Figure 1) and mycelium (Figure 1 and Supplementary Figure 1). Specific concentrations of lactic acid $[7.5 \%(\mathrm{v} / \mathrm{v})]$ inhibited but did not eliminate the growth of Monascus because the number of viable spores in the fermentation broth did not change much compared with the initial amount, and a similar result was observed when ethanol was added to $12.0 \%(\mathrm{v} / \mathrm{v})$. The inhibition of spore activity of Monascus by $7.5 \%(\mathrm{v} / \mathrm{v})$ lactic acid did not depend exclusively on $\mathrm{H}^{+}$concentration because the $\mathrm{pH}$ of this medium was 2.1 (Supplementary Figure 2), and Monascus was found to tolerate $10^{-1.5} \mathrm{~mol} / \mathrm{L} \mathrm{HCl}(\mathrm{pH}=1.5)$.

BHQ33 Hongqu powder was added to the medium containing different growth regulatory factors for 7 days (Figure 2). After fermentation, the fermentation broth was diluted and coated in modified PDA for observation. Coating results showed that adding $\mathrm{HCl}$ or ethanol alone did not help to obtain Monascus, while adding 4.5\% (v/v) lactic acid caused Monascus to appear in the plate. However, lactic acid alone did not inhibit Aspergillus niger, which grows significantly faster than Monascus, a situation that was unfavorable for obtaining as many different kinds of Monascus as possible. Combined with the results of Figures 2B3,C3, 9\% (v/v) ethanol was able to eliminate Aspergillus niger, while $4.5 \%(\mathrm{v} / \mathrm{v})$ lactic acid could eliminate yeast, and adding lactic acid and ethanol simultaneously was able to enrich Monascus (Figures 2D2,D3).

\section{Optimization of Lactic Acid and Ethanol Addition in the Restrictive Medium}

To eliminate fungi other than Monascus from BHQ33 Hongqu powder and to enrich as much Monascus as possible, response surface optimization was applied to optimize the amount of lactic acid and ethanol added to the restrictive medium. According to the tolerance test of Monascus in Figure 1, the concentration ranges of lactic acid and ethanol in the restrictive medium were selected to be $0 \sim 6 \%(\mathrm{v} / \mathrm{v})$ and $0 \sim 12 \%(\mathrm{v} / \mathrm{v})$, respectively. The response surface methodology uses a 2-factor 3-level model of Design-Expert software. The test scheme and results are listed in Table 1 and Figure 3. The test results in Table 1 confirm the conclusion in Figures 2C,D that it is difficult to grow Monascus only in culture dishes by using single lactic acid or ethanol. The fitting equation of the response surface model is as follows:

$$
\begin{aligned}
Y_{1}= & 4.64507+0.32330 A+0.01023 B-0.00458 A B \\
& -0.08453 A^{2}-0.00317 B^{2}
\end{aligned}
$$

$$
\begin{aligned}
Y_{2}= & 36.74284+24.53848 A+3.50424 B-0.38486 A B \\
& -2.42989 A^{2}-0.086916 B^{2}
\end{aligned}
$$

where $Y_{1}$ is the number of Monascus colonies, unit: Log10 $\mathrm{CFU} / \mathrm{mL} ; Y_{2}$ is the proportion of Monascus, unit: \%; $A$ is the amount of lactic acid added, unit: $\%(\mathrm{v} / \mathrm{v})$; and $B$ is the amount of ethanol added, unit: \% (v/v). The constraints of equations (1) and (2) are $f(A)=\min \{A\}, f(B)=\min \{B\}, f\left(Y_{1}\right)=\operatorname{man}\left\{Y_{1}\right\}$, $f\left(Y_{2}\right)=\operatorname{man}\left\{Y_{2}\right\}, 0 \leq \mathrm{A} \leq 6.0,0 \leq \mathrm{B} \leq 12.0$ and $Y_{2} \geq$ 100. The optimal solution is $\mathrm{A}=3.98, \mathrm{~B}=6.24, Y_{1}=4.420$, and $Y_{2}=104.8$ (according to the actual situation, $Y_{2}=100$ ).

Therefore, when the amount of lactic acid and ethanol reached $3.98 \%(\mathrm{v} / \mathrm{v})$ and $6.24 \%(\mathrm{v} / \mathrm{v})$, respectively, the amount of Monascus was $4.420 \log 10 \mathrm{CFU} / \mathrm{mL}$, and the proportion of Monascus was $100 \%$. The actual plate coating verification shows that the number of viable Monascus under this condition is $4.49 \pm 0.12 \log 10 \mathrm{CFU} / \mathrm{mL}$, accounting for $100 \%$ (3 strains of fungi could be obtained from the plate (Supplementary Figure 3), and all were Monascus purpureus after ITS gene sequencing).

\section{Dynamics of Monascus in Restrictive Medium During the Fermentation Process}

After the optimal addition of lactic acid and ethanol in the restrictive medium, the number and proportion of Monascus in the fermentation broth containing BHQ33 powder rapidly increased and gradually decreased from the first day to the 7 th day of fermentation (Figure 4A). However, the samples fermented to the 7th day did not affect the enrichment of Monascus, and the amount of Monascus still exceeded $1.0 \times 10^{4}$ $\mathrm{CFU} / \mathrm{mL}$, accounting for $100 \%$. To study the changing pattern of flora changes in this culture system more meticulously and determine the optimal enrichment duration, the enrichment process from 0 to $24 \mathrm{~h}$ was followed up by sampling and spread analysis (Figure 4B). The dominant fungi in the fermentation broth were Monascus, Saccharomyces cerevisiae, Aspergillus niger and Aspergillus flavus, while Lichtheimia ramos and Trichoderma were observed sporadically. From the initial state to $8 \mathrm{~h}$ of enrichment culture, the proportion of fungi except for Monascus gradually decreased until they disappeared. To obtain Monascus quickly and conveniently, sampling and coating are were carried 

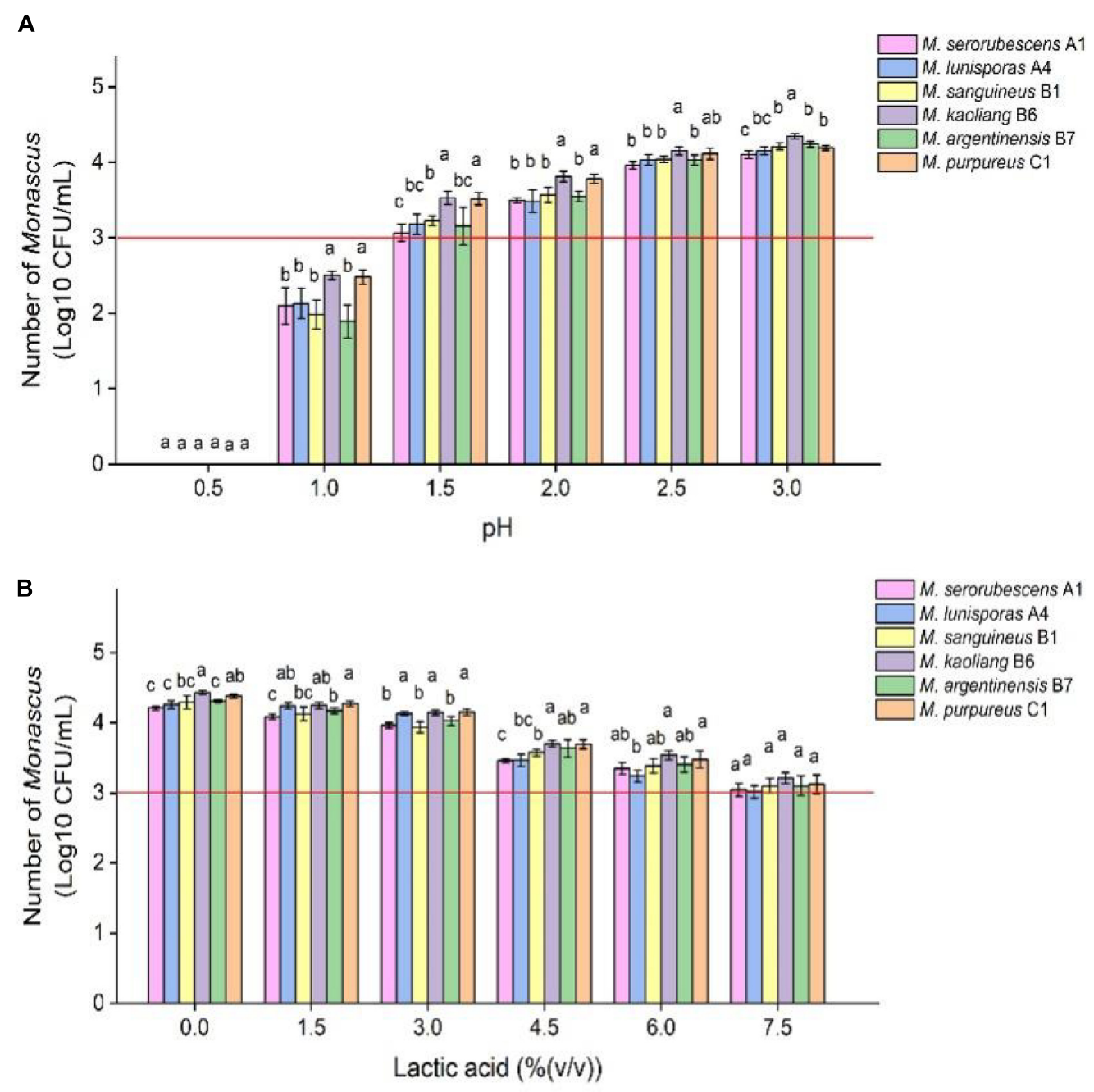

M. serorubescens A1

M. Junisporas A4

$M$. sanguineus $\mathrm{B} 1$

M. kaoliang $\mathrm{B} 6$

$M$. argentinensis $\mathrm{B} 7$

M. purpureus $\mathrm{C} 1$

C

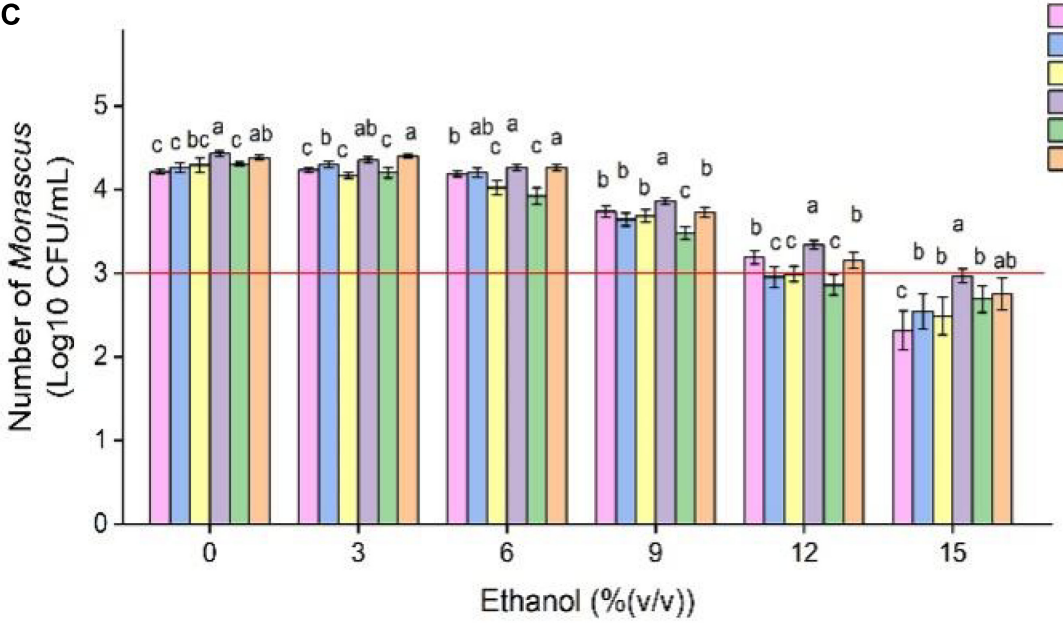

M. serorubescens A1

M. lunisporas A4

M. sanguineus $B 1$

M. kaoliang B6

$M$. argentinensis $\mathrm{B} 7$

M. purpureus $\mathrm{C} 1$

FIGURE 1 | Tolerance test of different species of Monascus to different growth inhibitors. The six Monascus species were cultured in restrictive medium containing different concentrations of $\mathrm{HCl}$ (A), lactic acid (B) and ethanol (C) at $30^{\circ} \mathrm{C}$ and $200 \mathrm{r} / \mathrm{min}$ for 7 days, and the number of viable spores of Monascus was determined by plate coating after fermentation. Error bars indicate the standard deviation (SD) of the means $(n=3)$, and different lowercase letters represent significant differences in the number of viable cells of different Monascus strains under the same treatment condition $(p<0.05)$. The red auxiliary line indicates that the initial amount of Monascus is $1.0 \times 10^{3} \mathrm{CFU} / \mathrm{mL}$. 


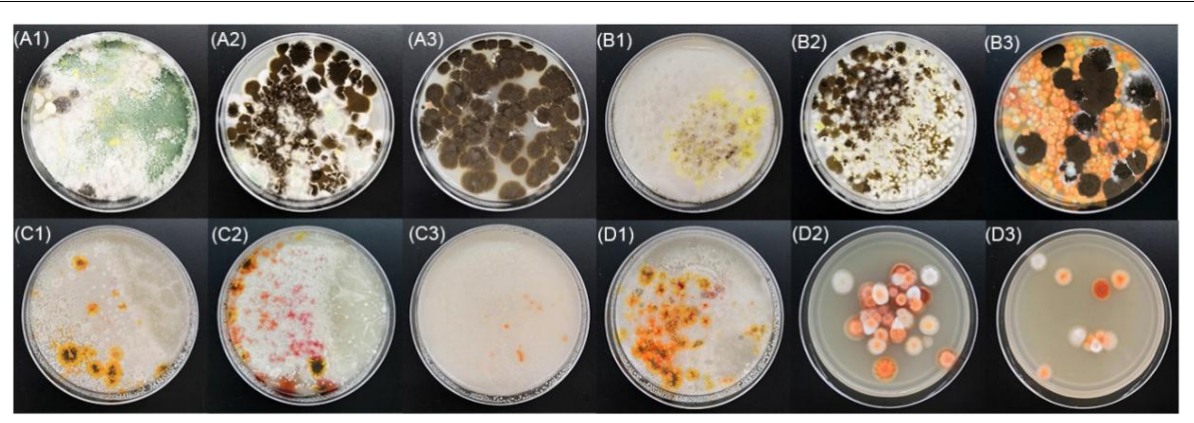

FIGURE 2 | Effect of different growth inhibitors on the enrichment of Monascus from Hongqu BHQ33. Hongqu BHQ33 was added to ferment respectively with different fermentation media containing $10^{-2.5} \mathrm{~mol} / \mathrm{L} \mathrm{HCl}$ (A1), $10^{-2} \mathrm{~mol} / \mathrm{L} \mathrm{HCl}$ (A2), $10^{-1.5} \mathrm{~mol} / \mathrm{L} \mathrm{HCl}$ (A3), $1.5 \%$ (v/v) lactic acid (B1), 3\% (v/v) lactic acid (B2), 4.5\% $(\mathrm{v} / \mathrm{v})$ lactic acid (B3), 3\% (v/v) ethanol (C1), 6\% (v/v) ethanol (C2), 9\% (v/v) ethanol (C3), 1.5\% (v/v) lactic acid \& 3\% (v/v) ethanol (D1), 3\% (v/v) lactic acid \& 6\% (v/v) ethanol (D2), $4.5 \%(\mathrm{v} / \mathrm{v})$ lactic acid \& $9 \%(\mathrm{v} / \mathrm{v})$ ethanol (D3) at $30^{\circ} \mathrm{C}$ and $200 \mathrm{r} / \mathrm{min}$ for 7 days, and then coated on the modified PDA medium with the fermentation broth to culture for 4 days at $30^{\circ} \mathrm{C}$.

out between 8 and $24 \mathrm{~h}$ after enrichment culture, which can save time and eliminate the interference of other fungi.

\section{Metabolic Behaviors of Monascus Against Lactic Acid and Ethanol Stress}

The fungi isolated from BHQ33 were inoculated into the restrictive medium containing different concentrations of lactic acid and ethanol for $24 \mathrm{~h}$ and sampled for analysis. Compared with experimental groups 1 and 2, the decreases in extracellular lactate and ethanol in experimental group 3 were significantly increased (Figures 5A,B), indicating that the cell membrane permeability could be changed by the synergistic effect of lactate and ethanol. Under the simultaneous stress of lactic acid and ethanol, Monascus reduced the amount of lactic acid in the cell,

TABLE 1 | Response surface test scheme and results for optimizing the addition of lactic acid and ethanol.

\begin{tabular}{|c|c|c|c|c|}
\hline Number & $\begin{array}{c}\text { Lactic } \\
\operatorname{acid}(\%(v / v))\end{array}$ & $\begin{array}{l}\text { Ethanol } \\
(\%(v / v))\end{array}$ & $\begin{array}{l}\text { The viable } \\
\text { count of } \\
\text { Monascus } \\
\text { (Log10 } \\
\text { CFU/mL) }\end{array}$ & $\begin{array}{c}\text { The } \\
\text { proportion of } \\
\text { Monascus (\%) }\end{array}$ \\
\hline 1 & 3 & 6 & 4.758 & 100 \\
\hline 2 & 0 & 12 & 4.271 & 68.77 \\
\hline 3 & 3 & 12 & 4.448 & 100 \\
\hline 4 & 0 & 0 & 4.668 & 36.42 \\
\hline 5 & 3 & 6 & 4.771 & 100 \\
\hline 6 & 6 & 12 & 2.824 & 100 \\
\hline 7 & 6 & 0 & 3.551 & 95.36 \\
\hline 8 & 3 & 6 & 4.678 & 100 \\
\hline 9 & 0 & 6 & 4.609 & 52.47 \\
\hline 10 & 3 & 0 & 4.822 & 89.95 \\
\hline 11 & 3 & 6 & 4.655 & 100 \\
\hline 12 & 3 & 6 & 4.671 & 100 \\
\hline 13 & 6 & 6 & 3.368 & 100 \\
\hline
\end{tabular}

Three parallel experiments were conducted in each group. For the convenience of software operation, only the mean value is input. and the intracellular scavenging ability of lactic acid and ethanol was better than that of other fungi (Figures 5C,D).

Extracellular metabolites were analyzed (Figure 6). For the experimental groups supplemented with lactic acid (groups 1 and 3), the amylase activity and extracellular reducing sugar concentration of Monascus were higher than those of other fungi, and their extracellular soluble starch contents and those of other filamentous fungi were not significantly different. Extracellular amylase can peel soluble starch from rice flour granules and then degrade soluble starch into reducing sugars, so it can be speculated that the liquefaction capacity and saccharification capacity of Monascus under lactic acid stress are better than those of other fungi, while higher contents of reducing sugars can provide more energy sources for cells.

Intracellular metabolites were analyzed (Figure 7). Lactic acid and ethanol can cause lipid peroxidation damage of cell membrane, so the content of MDA in experimental group 1 and experimental group 2 is higher than that in the control group. Lipid peroxidative damage to the cell membrane was more intense when lactic acid and ethanol were present simultaneously (Figure 7A). Under the synergistic stress of lactate and ethanol, the accumulated amount of MDA in Monascus cells was significantly lower than that in other fungi, which might be related to the higher activities of intracellular antioxidant enzymes (SOD, GSH and CAT). This antioxidant enzyme is able to scavenge oxygen free radicals and reduce the oxidative damage of polyunsaturated fatty acids in biofilms, which has a positive effect on maintaining the structure and function of biofilms.

\section{DISCUSSION}

In addition to a large number of Monascus, Hongqu also contains lactic acid bacteria, yeasts, and others (Liu et al., 2018; Hong et al., 2020). In the process of applying Hongqu to brew rice wine, it was found that Monascus has a certain resistance to lactic acid and alcohol (He et al., 2016; Cai et al., 2019; Liang et al., 2020), while the resistance of other microorganisms in the system is different from it (Lv et al., 2015; 

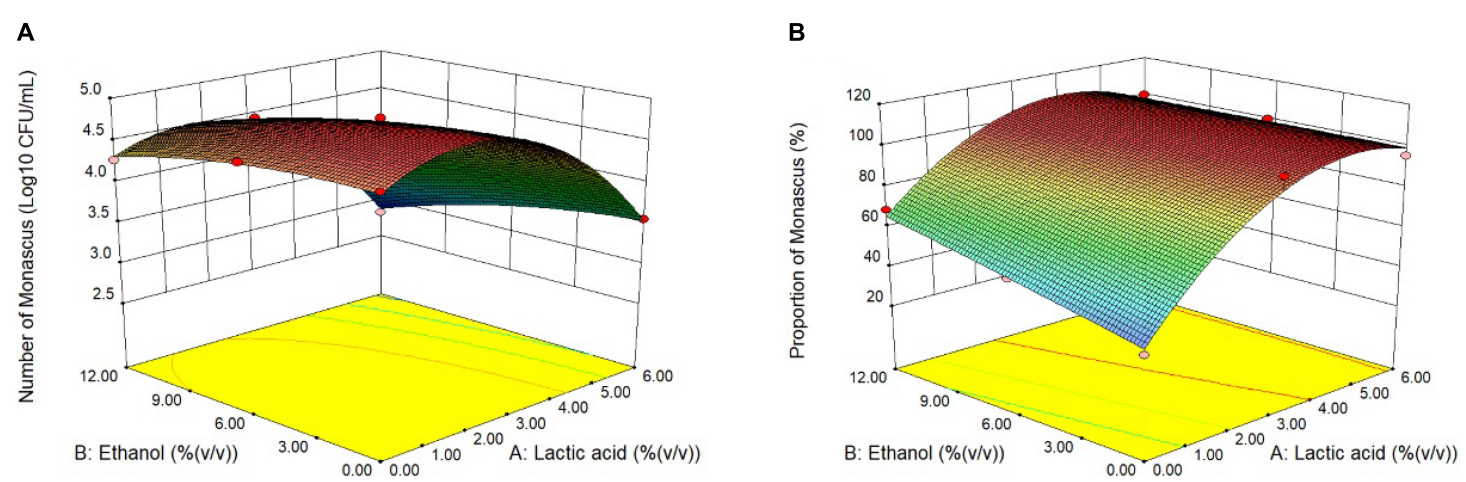

FIGURE 3 | Response surface optimization of lactic acid and ethanol addition in restrictive medium. Hongqu BHQ33 was added to the restrictive medium containing different concentrations of lactic acid and ethanol for fermentation at $30^{\circ} \mathrm{C}$ and $200 \mathrm{r} / \mathrm{min}$ for 7 days, the number of viable Monascus spores (A) and the proportion of the number of Monascus (B) were calculated after the fermentation broth was diluted and spread on the modified PDA medium to culture for 4 days at $30^{\circ} \mathrm{C}$.
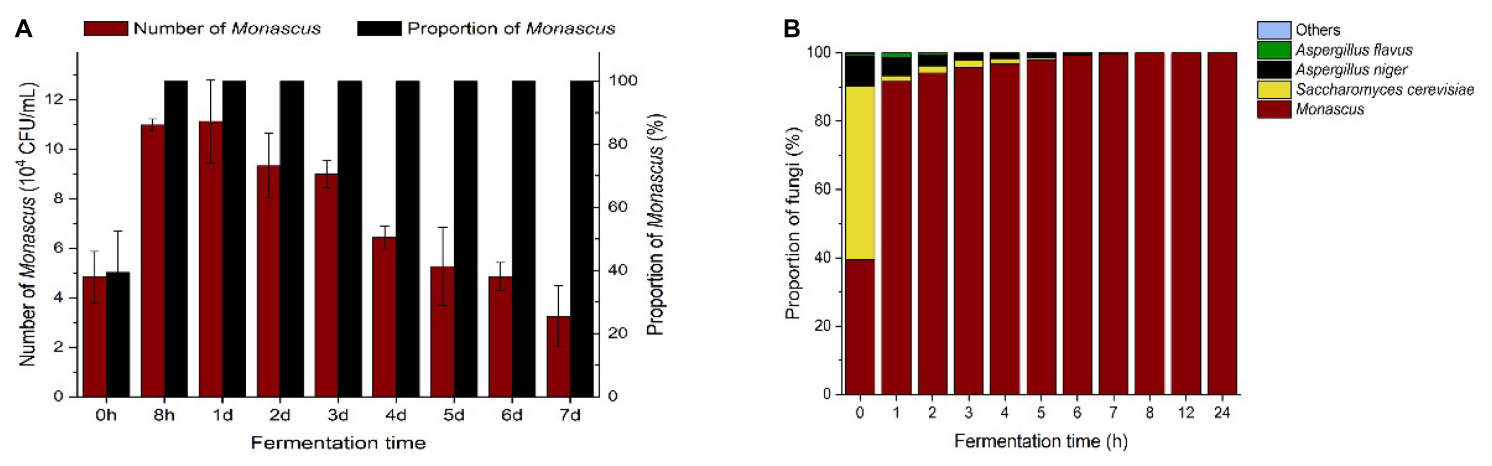

FIGURE 4 | Trends of Monascus and other culturable dominant fungi in the optimized restrictive medium supplemented with Hongqu BHQ33 during the fermentation process. Hongqu BHQ33 was added to the optimized restrictive medium containing 3.98\% (v/v) lactic acid and 6.24\%(v/v) ethanol for fermentation at $30^{\circ} \mathrm{C}$ and $200 \mathrm{r} / \mathrm{min}$ for 7 days, the number of Monascus and its proportion over time (A) and proportion of culturable dominant fungi over time (B) were calculated after the fermentation broth was diluted and spread on the modified PDA medium to culture for 4 days at $30^{\circ} \mathrm{C}$. Error bars indicate the standard deviation (SD) of the means $(n=3)$.

Huang et al., 2018). We speculate that it's a potential way to screen Manascus using the synergistic effect of lactic acid and ethanol, so finding the right ratio and concentration of both is the key point. In this study, when $4.5 \%(\mathrm{v} / \mathrm{v})$ lactic acid was added alone, Monascus and Aspergillus niger coexisted; while 9\% (v/v) ethanol was added alone, Monascus and Saccharomyces cerevisiae coexisted. After further optimization by response surface method, Monascus could be successfully enriched only when $3.98 \%(\mathrm{v} / \mathrm{v})$ lactic acid and $6.24 \%(\mathrm{v} / \mathrm{v})$ ethanol were added to the enrichment medium simultaneously. Traditionally, growth regulatory factors have been used to screen microbial strains, but they also may lead to the targeted domestication of the target strain, resulting in the loss of some metabolic functions (Yang et al., 2018). Fortunately, the novel restrictive medium for Monascus enrichment based on the synergistic stress of lactic acid and ethanol can shorten the pretreatment time without repeated operations, avoiding the abnormal changes in the metabolic characteristics of Monascus progeny to some extent. The morphological characteristics and metabolic function of Monascus progeny were not significantly changed after the treatment with the developed restrictive medium based on the synergistic stress of lactic acid and ethanol (Supplementary Figure 4 and Supplementary Table 1).

When cells are stimulated or stressed, the metabolic activities related to environmental adaptation become active, especially those related to substance transport, substance transformation, and energy metabolism processes. According to previous studies, lactic acid bacteria can resist the damage of acid to cells through neutralization process, biofilm and cell density, proton pump, protection of macromolecules, preadaptation and crossprotection, and effect of solutes (Wang et al., 2018); yeast can resist the damage of ethanol to cells through modification of the membrane, amino acids, trehalose and vacuolar protontranslocating ATPase functions (Saini et al., 2018). However, the tolerance mechanism of Monascus to the synergistic stress of lactic acid and ethanol has not been reported. The cell membrane is the key site to defend against environmental growth inhibitors, while the protection mechanism of the cell membrane is related to the kind and proportion of unsaturated fatty acids on the membrane because these fatty acids directly affect the fluidity of the phospholipid bilayer and the running capacity of carrier proteins on the membrane (Li et al., 2011; 

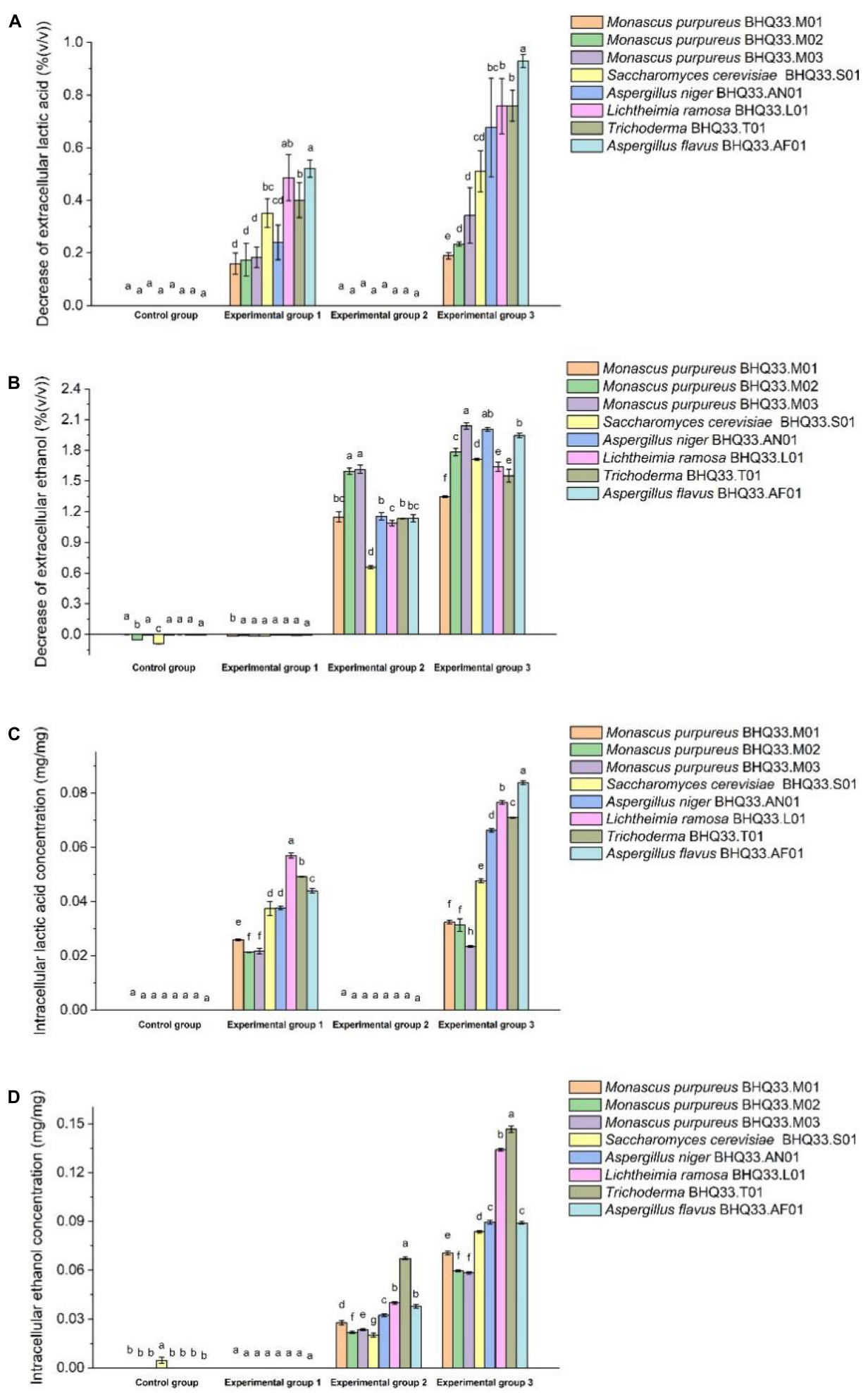

FIGURE 5 | Changes in intracellular and extracellular lactic acid and ethanol contents before and after fermentation by different fungi isolated from Hongqu BHQ33. Different fungi isolated from Hongqu $\mathrm{BHQ} 33$ were cultured in medium containing different concentrations of lactic acid and ethanol at $30^{\circ} \mathrm{C}$ and $200 \mathrm{r} / \mathrm{min}$ for $24 \mathrm{~h}$, and the decrease of extracellular lactic acid (A), the decrease of extracellular ethanol (B), the accumulation of intracellular lactic acid (C) and the accumulation of intracellular ethanol (D) were detected. In the initial medium, control group was basal medium without lactate and ethanol, experimental group 1 was supplemented with 3.98\% (v/v) lactic acid in basal medium, experimental group 2 with 6.24\% (v/v) ethanol, and experimental group 3 with both $3.98 \%$ (v/v) lactic acid and $6.24 \%$ $(\mathrm{v} / \mathrm{v})$ ethanol. Error bars indicate the standard deviation (SD) of the means $(n=3)$, and different lowercase letters represent significant differences in the detection indexes of fungi isolated from Hongqu BHQ33 under the same treatment conditions $(p<0.05)$. 


\section{A}

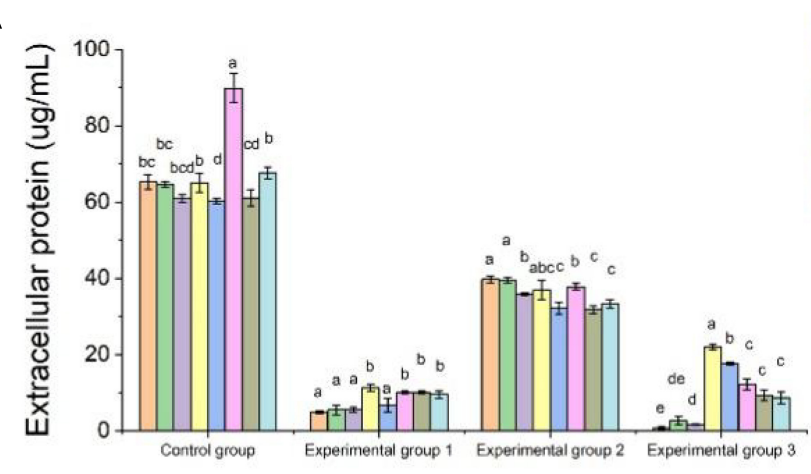

B

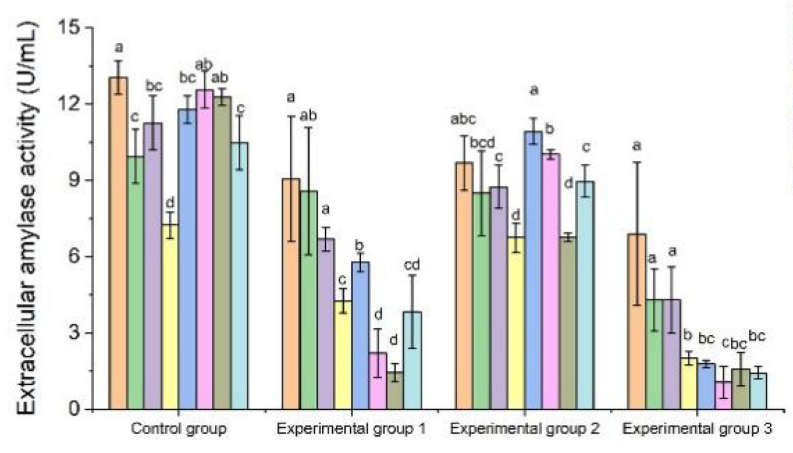

C

D

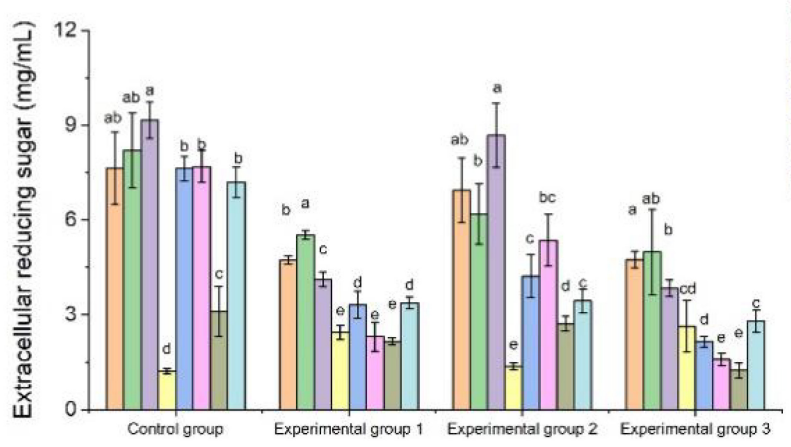

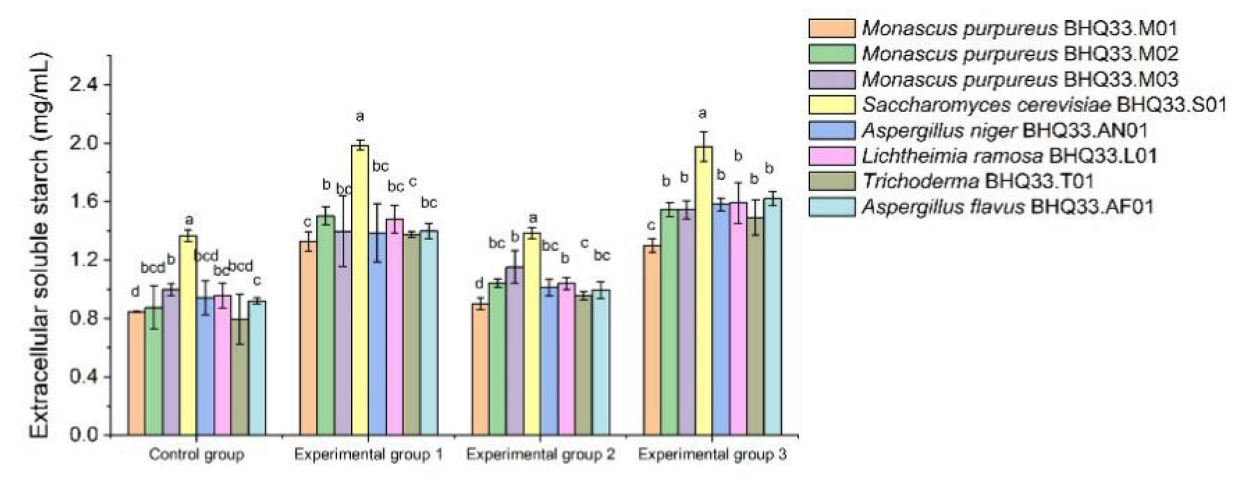

Monascus purpureus $\mathrm{BHQ} 33 \mathrm{MO} 1$

Monascus purpureus BHQ33.MO2

Monascus purpureus BHQ33.MO3

Saccharomyces cerevisiae BHQ33.S01

Aspergillus niger BHQ33.AN01

Lichtheimia ramosa BHQ33.L01

Trichoderma BHQ33.T01

Aspergillus flavus BHQ33.AF01

Monascus purpureus BHQ33.M01 Monascus purpureus BHQ33.M02 Cus purpureus $\mathrm{BHQ} 33 . \mathrm{MO} 3$

Saccharomyces cerevisiae BHQ33.SO

Aspergillus niger $\mathrm{BHQ} 33 . \mathrm{AN} 01$

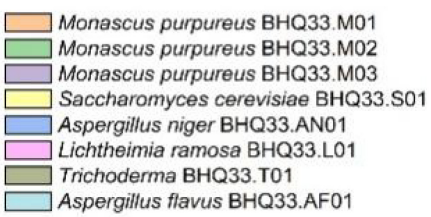

Monascus purpureus BHQ33.M01 Monascus purpureus BHQ33.MO2

Saccharomyces cerevisiae BHQ33.S01

Aspergillus niger BHQ33.AN01

Trichoderma BHQ33.T01

Aspergillus flavus BHQ33.AF01

FIGURE 6 | Degradation and utilization of substrates by different fungi isolated from Hongqu BHQ33. Different fungi isolated from Hongqu BHQ33 were cultured in medium containing different concentrations of lactic acid and ethanol at $30^{\circ} \mathrm{C}$ and $200 \mathrm{r} / \mathrm{min}$ for $24 \mathrm{~h}$, and extracellular protein concentration (A), extracellular amylase activity (B), extracellular soluble starch concentration (C), and extracellular reducing sugar concentration (D) were detected. In the initial medium, control group was basal medium without lactate and ethanol, experimental group 1 was supplemented with $3.98 \%(\mathrm{~V} / \mathrm{V})$ lactic acid in basal medium, experimental group 2 with $6.24 \%(\mathrm{~V} / \mathrm{v})$ ethanol, and experimental group 3 with both $3.98 \%(\mathrm{~V} / \mathrm{V})$ lactic acid and $6.24 \%(\mathrm{~V} / \mathrm{V})$ ethanol. Error bars indicate the standard deviation (SD) of the means $(n=3)$, and different lowercase letters represent significant differences in the detection indexes of fungi isolated from Hongqu BHQ33 under the same treatment conditions $(p<0.05)$. 

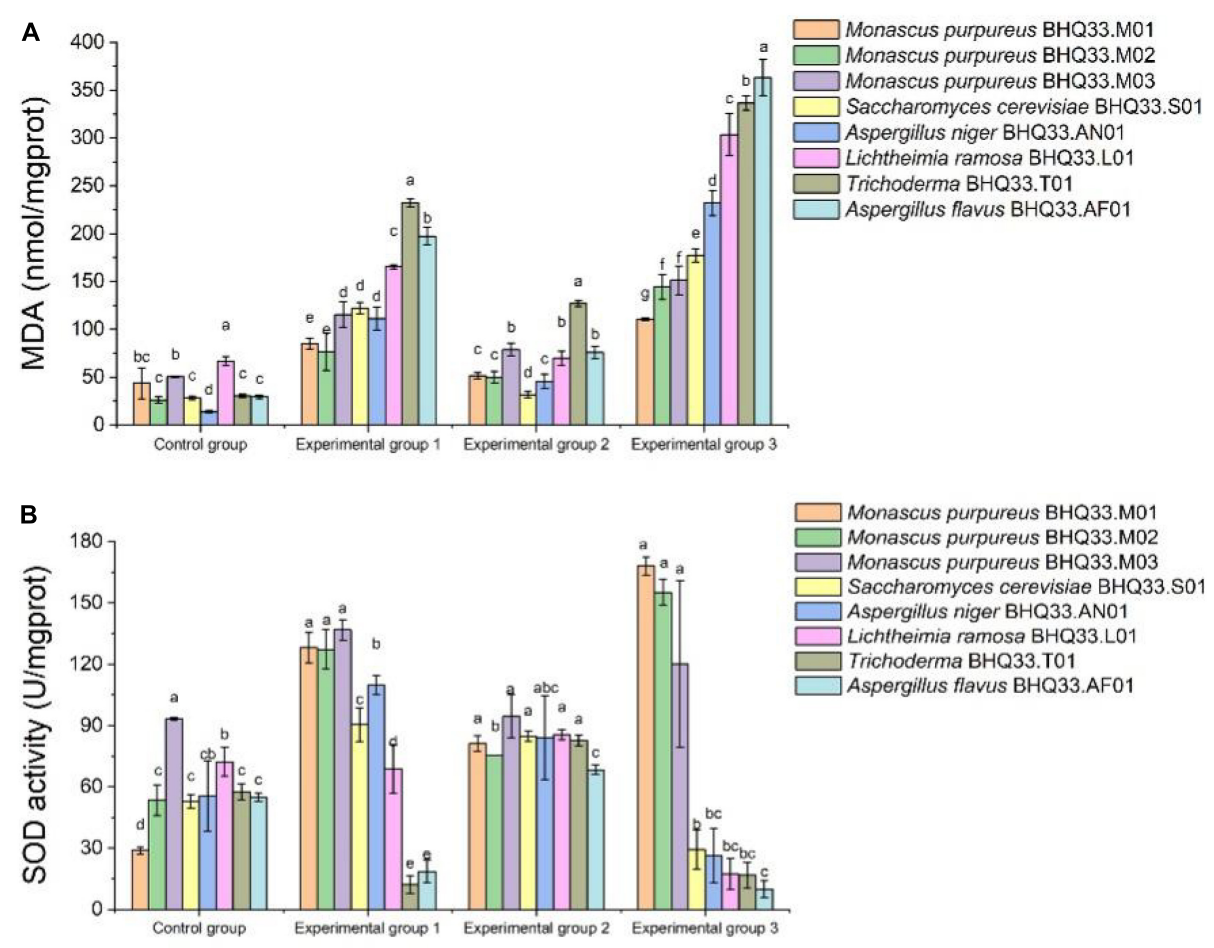

Monascus purpureus BHQ33.M01

Monascus purpureus BHQ33.M02 Monascus purpureus BHQ33.M03

Saccharomyces cerevisiae BHQ33 S01

Aspergillus niger BHQ33.ANO1

Lichtheimia ramosa BHQ33. L01

Trichoderma BHQ33.T01

Aspergillus flavus BHQ33.AF01

\section{C}

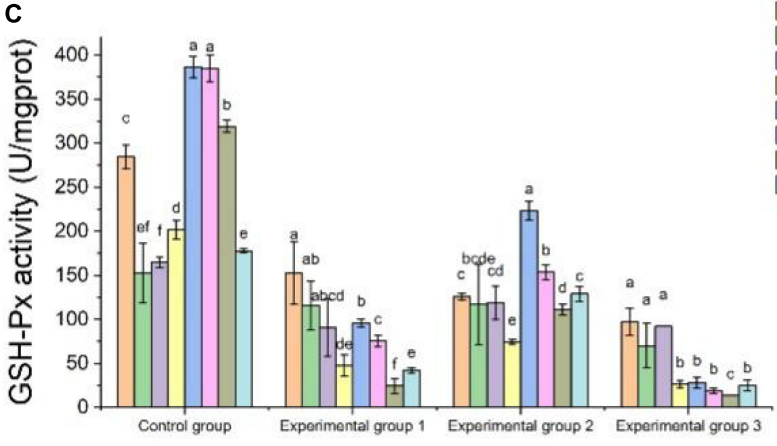

$\square$ Monascus purpureus BHQ33.M01

Monascus purpureus $\mathrm{BHQ} 33 . \mathrm{MO} 2$

Monascus purpureus BHQ33.M03

Saccharomyces cerevisiae BHQ33.S01

Aspergillus niger BHQ33.ANO1

Lichtheimia ramosa BHQ33.L01

Trichoderma BHQ33.T01

Aspergillus flavus BHQ33.AF01

D

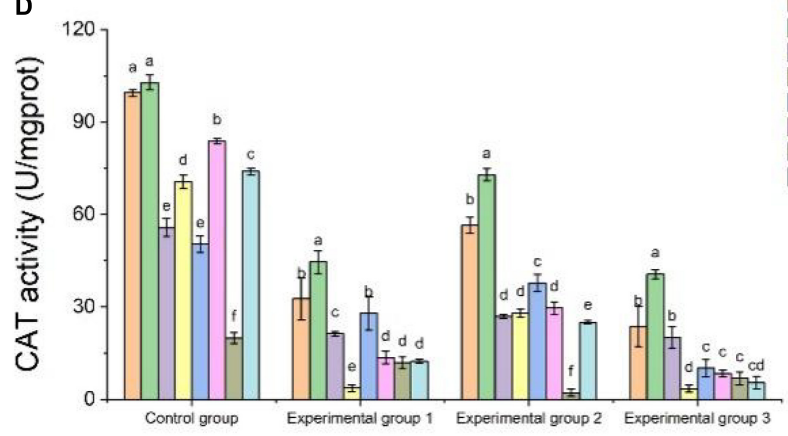

Monascus purpureus BHQ33.M01

Monascus purpureus BHQ33.M02

Monascus purpureus BHQ33.M03

Saccharomyces cerevisiae BHQ33.S01

Aspergillus niger BHQ33.AN01

Lichtheimia ramosa BHQ33.L01

Trichoderma BHQ33.T01

Aspergillus flavus BHQ33.AF01

Experimental group 1 Experimental group 2 Experimental group 3

FIGURE 7 | Intracellular antioxidant capacity of different fungi isolated from Hongqu BHQ33 under lactic and ethanol stress. Different fungi isolated from Hongqu $\mathrm{BHQ} 33$ were cultured in medium containing different concentrations of lactic acid and ethanol at $30^{\circ} \mathrm{C}$ and $200 \mathrm{r} / \mathrm{min}$ for $24 \mathrm{~h}$, and intracellular metabolites malondialdehyde (MDA) (A), total superoxide dismutase (SOD) (B), total glutathione peroxidase (GSH-Px) (C), and catalase (CAT), (D) were detected. In the initial medium, control group was basal medium without lactate and ethanol, experimental group 1 was supplemented with $3.98 \%$ (v/v) lactic acid in basal medium, experimental group 2 with $6.24 \%$ (v/v) ethanol, and experimental group 3 with both $3.98 \%(\mathrm{v} / \mathrm{v}$ ) lactic acid and $6.24 \%$ (v/v) ethanol. Error bars indicate the standard deviation (SD) of the means ( $n=3$ ), and different lowercase letters represent significant differences in the detection indexes of fungi isolated from Hongqu BHQ33

under the same treatment conditions $(p<0.05)$. 


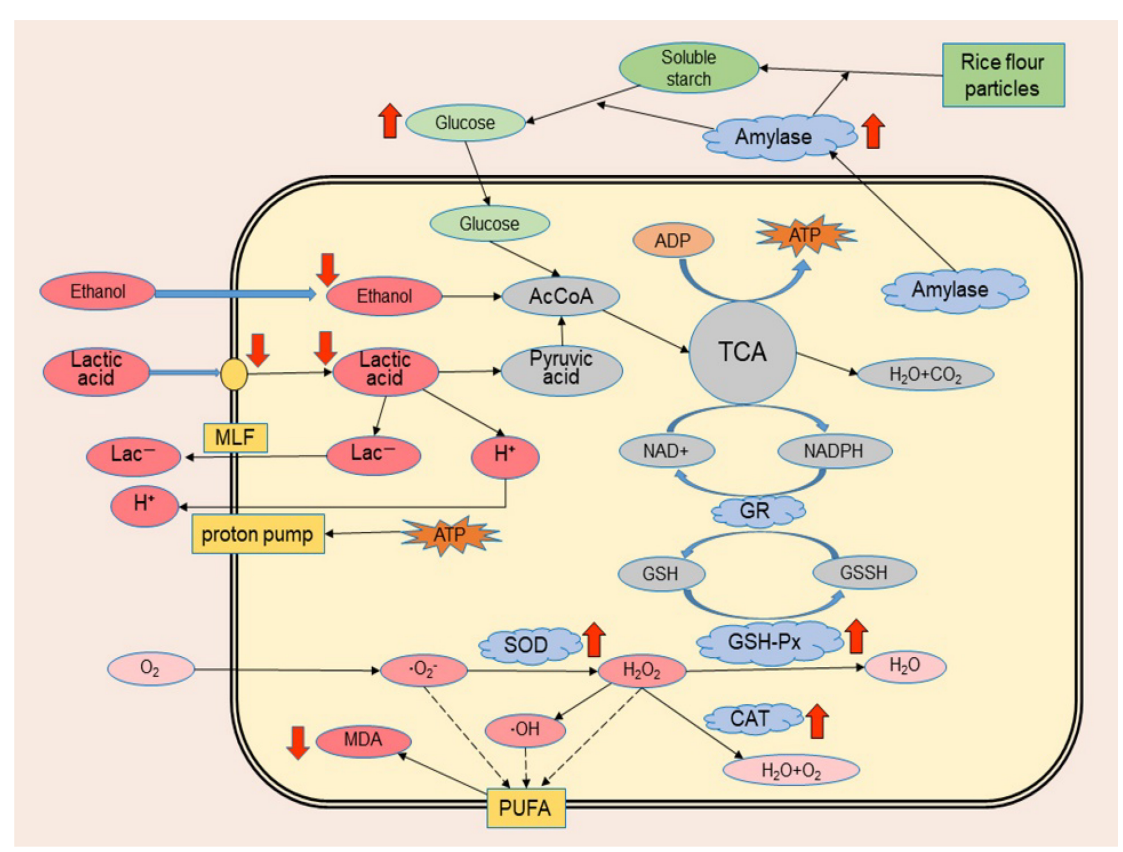

FIGURE 8 | Schematic illustration of the possible tolerance mechanism of Monascus to synergistic stress of lactic acid and ethanol. Red arrows indicate significant metabolic differences between Monascus and other fungi isolated from BHQ33 in the optimized restrictive medium containing both lactic acid and ethanol, with arrows up-regulated and arrows down-regulated.

Fonseca et al., 2019; Liu et al., 2020). For example, the addition of oleic acid to lipid-poor media significantly reduced oxidative damage to Saccharomyces cerevisiae cell membranes and resulted in improved resistance to oxidative stress (Landolfo et al., 2010). In addition, lactic acid and ethanol can cause oxidative damage to cells. Excessive reactive oxygen species will attack unsaturated fatty acids on the cell membrane, resulting in increased intracellular MDA, which may cause cross-linking polymerization of life macromolecules such as proteins and nucleic acids and have a toxic effect on cells (Talbi et al., 2019; Požgajová et al., 2020). The oxidative damage of unsaturated fatty acids also affects the resistance of the cell membrane to external growth inhibitors (Fonseca et al., 2019; Liu et al., 2020). Monascus has an active system of fatty acid metabolism (Peters et al., 1993; Moharram et al., 2012). The polyketide synthesis (pigment, lovastatin and citrinin) of Monascus are associated with fatty acid metabolism which also provides unsaturated long-chain fatty acids to serve as synthetic feedstocks for phospholipids on biological membranes (Wang, 2006; Panda and Ali, 2012; Balakrishnan et al., 2014; Thomanek et al., 2018). Therefore, it can be speculated that the simultaneous tolerance of Monascus to lactic acid and ethanol may be associated with the protective function of cell membranes, and this series of metabolic processes is involved in maintaining the structure and function of cell membranes. In the presence of higher concentrations of lactic acid and ethanol simultaneously, Monascus is more resistant to cell invasion by lactic acid than other fungi and alleviates the interference of lactic acid and ethanol on the normal metabolism of cells; intracellular antioxidant enzymes are able to reduce the oxidative damage of unsaturated fatty acids on the cell membrane; and higher activity of extracellular amylase and higher concentrations of extracellular reducing sugars are able to provide energy for the cell to defend against stress (Figure 8).

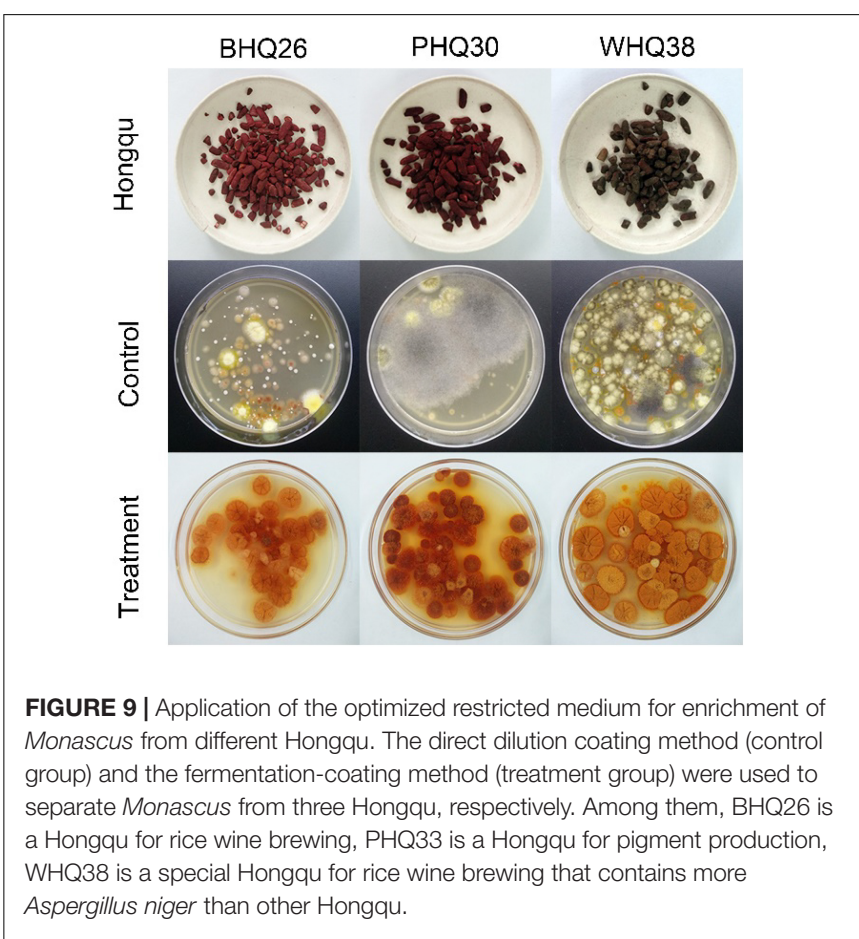


It is worth mentioning that an attempt to prejudge by highthroughput sequencing how many kinds of Monascus species are present in the Hongqu BHQ33 revealed only Monascus purpureus in this sample (Supplementary Figure 5), but it is not clear how many different types of Monascus exist. This study obtained 3 strains of Monascus purpureus with different morphological types by using optimized restricted medium supplemented with lactic acid and ethanol (Supplementary Figure 3), which illustrated that a suitable culture method was able to increase the discrimination ability of Monascus species and subspecies levels and could also assist in analyzing the species distribution of Monascus in Hongqu at the species level.

Finally, this method was applied to Monascus enrichment from different types of Hongqu (Figure 9). Although the fungi in these Hongqu are very complex and diverse, Monascus pure culture can still be enriched and obtained by the restrictive medium based on the synergistic stress of lactic acid and ethanol. The feasibility of lactic acid and ethanol as regulators for directional enrichment of Monascus was verified. The possible mechanisms on the tolerance of Monascus to the synergistic stress of lactic acid and ethanol can be further explored by transcriptomics and metabolomics, which has important practical significance for the application of Monascus in fermentation.

\section{CONCLUSION}

The preparation of Monascus pure culture from Hongqu is easily interfered by other microorganisms. Therefore, the development of restrictive medium for Monascus enrichment from Hongqu is of great significance for the screening of excellent Monascus strains. This study showed that Monascus has good tolerance to lactic acid and ethanol, and retains the vitality of spore germination at high concentrations of lactic acid and ethanol. Under the synergistic stress of lactic acid and ethanol, Monascus pure culture can be directionally enriched from Hongqu at appropriate concentrations without affecting the morphological characteristics and metabolic function in Monascus progeny.

\section{REFERENCES}

Agboyibor, C., Kong, W., Chen, D., Zhang, A., and Niu, S. (2018). Monascus pigments production, composition, bioactivity and its application: a review. Biocatal. Agric. Biotechnol. 16, 433-447. doi: 10.1016/j.bcab.2018.09.012

Ai, M., Qiu, X., Huang, J., Wu, C., Jin, Y., and Zhou, R. (2019). Characterizing the microbial diversity and major metabolites of Sichuan bran vinegar augmented by Monascus purpureus. Int. J. Food Microbiol. 292, 83-90. doi: 10.1016/j. ijfoodmicro.2018.12.008

Balakrishnan, B., Kim, H. J., Suh, J. W., Chen, C. C., Liu, K. H., Park, S. H., et al. (2014). Monascus azaphilone pigment biosynthesis employs a dedicated fatty acid synthase for short chain fatty acyl moieties. J. Korean Soc. Appl. Biol. Chem. 57, 191-196. doi: 10.1007/s13765-014-4017-0

Balia, R. L., Kurnani, T. B. A., and Utama, G. L. (2018). Selection of mozzarella cheese whey native yeasts with ethanol and glucose tolerance ability. Int. J. Adv. Sci. Eng. Inf. Technol. 8, 1091-1097. doi: 10.18517/ijaseit.8.4.5869

Barbosa, R. N., Leong, S. L., Vinnere-Pettersson, O., Chen, A. J., Souza-Motta, C. M., Frisvad, J. C., et al. (2017). Phylogenetic analysis of Monascus and new species from honey, pollen and nests of stingless bees. Stud. Mycol. 86, 29-51. doi: 10.1016/j.simyco.2017.04.001
Monascus can maintain the barrier function of of its cell membrane and help it defend against the synergistic stress of lactic acid and ethanol. The novel restrictive medium developed for Monascus enrichment from Hongqu based on the synergistic stress of lactic acid and ethanol is of great value for the construction of Monascus strain libraries and better development of their germplasm resources.

\section{DATA AVAILABILITY STATEMENT}

The datasets presented in this study can be found in online repositories. The names of the repository/repositories and accession number(s) can be found in the article/ Supplementary Material.

\section{AUTHOR CONTRIBUTIONS}

KZ, XL, and LN designed the experiments. KZ, LW, GC, and $\mathrm{XZ}$ carried out the experiments. $\mathrm{KZ}$, WZ, and $\mathrm{CZ}$ analyzed the experimental results. $\mathrm{KZ}, \mathrm{XL}, \mathrm{LN}, \mathrm{ZL}$, and $\mathrm{PR}$ wrote the manuscript. All authors contributed to the article and approved the submitted version.

\section{FUNDING}

This work was supported by National Keypoint Research and Invention Program of the Thirteenth (No. 2018YFC1604106), University-Industry Cooperation Project of the Science and Technology Department of Fujian Province (No. 2019N5002).

\section{SUPPLEMENTARY MATERIAL}

The Supplementary Material for this article can be found online at: https://www.frontiersin.org/articles/10.3389/fmicb. 2021.702951/full\#supplementary-material

Cai, Q., Zhou, K., Liu, Z., Zhang, C., Zhang, W., and Ni, L. (2019). Studies on growth inhibitory factors of Monascus in the brewing process of Hongqu rice wine. J. Chin. Inst. Food Sci. Technol. 19, 143-149. doi: 10.16429/j.1009-7848. 2019.10.017

Chysirichote, T., Takahashi, R., Asami, K., and Ohtaguchi, K. (2013). Effects of starch and protein on glucosamine content in the biomass of Monascus ruber. J. Chem. Eng. Japan 46, 695-698. doi: 10.1252/jcej.13we026

Eren, T., Atar, N., Yola, M. L., and Karimi-Maleh, H. (2015). A sensitive molecularly imprinted polymer based quartz crystal microbalance nanosensor for selective determination of lovastatin in red yeast rice. Food Chem. 185, 430-436. doi: 10.1016/j.foodchem.2015.03.153

Fonseca, F., Pénicaud, C., Tymczyszyn, E. E., Gómez-Zavaglia, A., and Passot, S. (2019). Factors influencing the membrane fluidity and the impact on production of lactic acid bacteria starters. Appl. Microbiol. Biotechnol. 103, 6867-6883. doi: 10.1007/s00253-019-10002-1

He, D., Liang, S., Liu, Z., Zhang, W., and Ni, L. (2016). Application of liquid-state Hong Qu to the brewing of Fujian Hong Qu glutinous rice wine. J. Chin. Inst. Food Sci. Technol. 16, 133-140. doi: 10.16429/j.1009-7848.2016.12.018

Hong, J., Chen, M., Zhou, W., Guo, W., Li, L., Huang, Z., et al. (2020). Effects of lactic acid bacteria on the growth of yeast and the formation of flavor 
compounds in the fermentation of Hongqu glutinous rice wine. J. Chin. Inst. Food Sci. Technol. 20, 91-100. doi: 10.16429/j.1009-7848.2020.01.012

Huang, C., Wu, L., Lu, L., Zhang, Z., and Liu, Z. (2014). Isolation and identification of Monascus sp. strain with monacolin K producing ability from Guangxi red yeast rice. China Condiment 39, 36-40. doi: 10.3969/j.issn.1000-9973.2014.05. 010

Huang, Z., Guo, W., Zhou, W., Li, L., Xu, J., Hong, J., et al. (2019). Microbial communities and volatile metabolites in different traditional fermentation starters used for Hong Qu glutinous rice wine. Food Res. Int. 121, 593-603. doi: 10.1016/j.foodres.2018.12.024

Huang, Z., Hong, J., Xu, J., Li, L., Guo, W., Pan, Y., et al. (2018). Exploring core functional microbiota responsible for the production of volatile flavour during the traditional brewing of Wuyi Hong Qu glutinous rice wine. Food Microbiol. 76, 487-496. doi: 10.1016/j.fm.2018.07.014

Jiang, Y., Lv, X., Zhang, C., Zheng, Y., Zheng, B., Duan, X., et al. (2019). Microbial dynamics and flavor formation during the traditional brewing of Monascus vinegar. Food Res. Int. 125:108531. doi: 10.1016/j.foodres.2019.108531

Landolfo, S., Zara, G., Zara, S., Budroni, M., Ciani, M., and Mannazzu, I. (2010). Oleic acid and ergosterol supplementation mitigates oxidative stress in wine strains of Saccharomyces cerevisiae. Int. J. Food Microbiol. 141, 229-235. doi: 10.1016/j.ijfoodmicro.2010.05.020

Lei, M., Liu, J., Fang, Y., Shao, Y., Li, L., Yu, J. H., et al. (2019). Effects of different g-protein $\alpha$-subunits on growth, development and secondary metabolism of Monascus ruber M7. Front. Microbiol. 10:1555. doi: 10.3389/fmicb.2019.01555

Li, X., Wang, J., Bu, D., Wei, H., Hu, H., and Zhou, L. (2011). Advanced research of effect of polyunsaturated fatty acids on cell membrane function. Biotechnol. Bull. 12, 22-26.

Liang, Z., Lin, X., He, Z., Li, W., Ren, X., and Lin, X. (2020). Dynamic changes of total acid and bacterial communities during the traditional fermentation of Hong Qu glutinous rice wine. Electron. J. Biotechnol. 43, 23-31. doi: 10.1016/j. ejbt.2019.12.002

Liu, P., Zheng, X., Duan, C., Chen, B., and Yan, G. (2020). A review of the effect of unsaturated fatty acids on the cell growth and aroma production of Saccharomyces cerevisiae during fermentation. Food Sci. 41, 314-322. doi: 10.7506/spkx1002-6630-20190729-397

Liu, Z., Wang, Z., Lv, X., Zhu, X., Chen, L., and Ni, L. (2018). Comparison study of the volatile profiles and microbial communities of Wuyi Qu and Gutian Qu, two major types of traditional fermentation starters of Hong Qu glutinous rice wine. Food Microbiol. 69, 105-115. doi: 10.1016/j.fm.2017.07.019

Lv, X., Chen, Z., Jia, R., Liu, Z., Wen, Z., Shaojun, C., et al. (2015). Microbial community structure and dynamics during the traditional brewing of Fuzhou Hong Qu glutinous rice wine as determined by culture-dependent and cultureindependent techniques. Food Control 57, 216-224. doi: 10.1016/j.foodcont. 2015.03.054

Lv, X., Jia, R., Li, Y., Chen, F., Chen, Z., Liu, B., et al. (2016). Characterization of the dominant bacterial communities of traditional fermentation starters for Hong Qu glutinous rice wine by means of MALDI-TOF mass spectrometry fingerprinting, 16S rRNA gene sequencing and species-specific PCRs. Food Control 67, 292-302. doi: 10.1016/j.foodcont.2016.03.005

Lv, X., Weng, X., Han, M., Zhang, W., Rao, P., and Ni, L. (2012). Identification and characterization of Monascus sp. from Fujian Hongqu. J. Chin. Inst. Food Sci. Technol. 12, 88-97. doi: 10.16429/j.1009-7848.2012.02.019

Moharram, A. M., Mostafa, M. E., and Ismail, M. A. (2012). Chemical profile of Monascus ruber strains. Food Technol. Biotechnol. 50, 490-499. doi: 10.1016/j. ifset.2012.09.007

Panda, B. P., and Ali, M. (2012). Reduction of citrinin biosynthesis by fatty acids in Monascus fermented food. World Mycotoxin J. 5, 163-167. doi: 10.3920/ wmj2011.1335

Park, H. G., Stamenova, E. K., and Jong, S. C. (2004). Phylogenetic relationships of Monascus species inferred from the ITS and the partial b-tubulin gene. Bot. Bull. Acad. Sin. 47, 325-330. doi: 10.7016/BBAS.200410.0325

Park, K. H., Liu, Z., Park, C. S., and Ni, L. (2016). Microbiota associated with the starter cultures and brewing process of traditional Hong Qu glutinous rice wine. Food Sci. Biotechnol. 25, 649-658. doi: 10.1007/s10068-016-0115-6

Peters, N., Panitz, C., and Kunz, B. (1993). The influence of carbohydrate dissimilation on the fatty acid metabolism of Monascus purpureus. Appl. Microbiol. Biotechnol. 39, 589-592. doi: 10.1007/bf00205057

Požgajová, M., Navrátilová, A., Šebová, E., Kovár, M., and Kačániová, M. (2020). Cadmium-induced cell homeostasis impairment is suppressed by the torl deficiency in fission yeast. Int. J. Mol. Sci. 21:7847. doi: 10.3390/ijms21217847
Prasirtsak, B., Thitiprasert, S., Tolieng, V., Assabumrungrat, S., Tanasupawat, S., and Thongchul, N. (2017). Characterization of D-lactic acid, spore-forming bacteria and Terrilactibacillus laevilacticus SK5-6 as potential industrial strains. Ann. Microbiol. 67, 763-778. doi: 10.1007/s13213-017-1306-y

Ren, X., He, Z., Lin, X., Lin, X., Liang, Z., Liu, D., et al. (2021). Screening and evaluation of Monascus purpureus FJMR24 for enhancing the raw material utilization rate in rice wine brewing. J. Sci. Food Agric. 101, 185-193. doi: 10.1002/jsfa. 10630

Saini, P., Beniwal, A., Kokkiligadda, A., and Vij, S. (2018). Response and tolerance of yeast to changing environmental stress during ethanol fermentation. Process Biochem. 72, 1-12. doi: 10.1016/j.procbio.2018.07.001

Sakural, Y., Lee, H. G., and Shiota, H. (1977). On the convenient method for glucosamine estimation in koji. Agric. Biol. Chem. 41, 619-624. doi: 10.1080/ 00021369.1977.10862552

Shin, H. M., Lim, J. W., Shin, C. G., and Shin, C. S. (2017). Comparative characteristics of rice wine fermentations using Monascus koji and rice nuruk. Food Sci. Biotechnol. 26, 1349-1355. doi: 10.1007/s10068-017-0187-y

Talbi, W., Ghazouani, T., Braconi, D., Ben Abdallah, R., Raboudi, F., Santucci, A., et al. (2019). Effects of selenium on oxidative damage and antioxidant enzymes of eukaryotic cells: wine Saccharomyces cerevisiae. J. Appl. Microbiol. 126, 555-566. doi: 10.1111/jam.14150

Thomanek, N., Arends, J., Lindemann, C., Barkovits, K., Meyer, H. E., Marcus, K., et al. (2018). Intricate crosstalk between lipopolysaccharide, phospholipid and fatty acid metabolism in Escherichia coli modulates proteolysis of LpxC. Front. Microbiol. 9:3285. doi: 10.3389/fmicb.2018.03285

Tian, Y., Huang, J., Xie, T., Huang, L., Zhuang, W., Zheng, Y., et al. (2016). Oenological characteristics, amino acids and volatile profiles of Hongqu rice wines during pottery storage: effects of high hydrostatic pressure processing. Food Chem. 203, 456-464. doi: 10.1016/j.foodchem.2016.02.116

Wang, C., Cui, Y., and Qu, X. (2018). Mechanisms and improvement of acid resistance in lactic acid bacteria. Arch. Microbiol. 200, 195-201. doi: 10.1007/ s00203-017-1446-2

Wang, P. (2006). Cloning and Analysis of the Monacolin K cDNA Encoding Biosynthesis Related Genes in Monascus purpureus BCRC 31615. Master's thesis. Taibei: National Taiwan University.

Weusthuis, R. A., Mars, A. E., Springer, J., Wolbert, E. J., Wal, H. V. D., Vrije, T. G. D., et al. (2017). Monascus ruber as cell factory for lactic acid production at low pH. Metab. Eng. 42, 66-73. doi: 10.1016/j.ymben.2017.05.005

Xiong, Z., Cao, X., Wen, Q., Chen, Z., Cheng, Z., Huang, X., et al. (2019). An overview of the bioactivity of monacolin K / lovastatin. Food Chem. Toxicol. 131:110585. doi: 10.1016/j.fct.2019.110585

Xu, M., Lv, X., Liu, Z., Zhang, W., and Ni, L. (2014). Identification and characterization of Rhizopus sp. from Fujian White Koji. J. Chin. Inst. Food Sci. Technol. 14, 205-210. doi: 10.16429/j.1009-7848.2014.03.049

Yang, Y., Xia, Y., Lin, X., Wang, G., Zhang, H., Xiong, Z., et al. (2018). Improvement of flavor profiles in Chinese rice wine by creating fermenting yeast with superior ethanol tolerance and fermentation activity. Food Res. Int. 108, 83-92. doi: 10.1016/j.foodres.2018.03.036

Zhang, R., Li, Q., Zhu, L., Liu, C., and Li, Y.-X. (2018). Study on the $\gamma$-aminobutyric acid (GABA) in highland barley Monascus beer. J. Food Sci. Biotechnol. 37, 1148-1152. doi: 10.3969/j.issn.1673-1689.2018.11.005

Zhang, W., Cai, Q., Rao, T., Zhou, K., Huang, Y., Liu, Z., et al. (2015). Analysis of dominate fungal community and characteristics of Gutian Hong Qu. J. Chin. Inst. Food Sci. Technol. 15, 180-185. doi: 10.16429/j.1009-7848.2015.12.025

Zhou, W., Guo, R., Guo, W., Hong, J., Li, L., Ni, L., et al. (2019). Monascus yellow, red and orange pigments from red yeast rice ameliorate lipid metabolic disorders and gut microbiota dysbiosis in Wistar rats fed on a high-fat diet. Food Funct. 10, 1073-1084. doi: 10.1039/c8fo02192a

Conflict of Interest: The authors declare that the research was conducted in the absence of any commercial or financial relationships that could be construed as a potential conflict of interest.

Copyright (C) 2021 Zhou, Wu, Chen, Liu, Zhao, Zhang, Lv, Zhang, Rao and Ni. This is an open-access article distributed under the terms of the Creative Commons Attribution License (CC BY). The use, distribution or reproduction in other forums is permitted, provided the original author(s) and the copyright owner(s) are credited and that the original publication in this journal is cited, in accordance with accepted academic practice. No use, distribution or reproduction is permitted which does not comply with these terms. 